\title{
Clinical development of therapeutic recombinant proteins
}

\author{
Janice M. Reichert and Cherie Paquette
}

BioTechniques 35:176-185 (July 2003)

\begin{abstract}
Only a small subset of the therapeutics that enter clinical studies will prove to be safe and effective in humans and gain approval for marketing. The success of the products and, by inference, the sponsoring companies can be measured by tracking advancement through the clinical phase and review transitions to marketing approval. To determine phase transition probabilities and approval success rates for recombinant protein (rDNA) therapeutics, the Tufts Center for the Study of Drug Development collected data for 271 rDNA therapeutics that entered clinical study between 1980 and 2002. The data were stratified into eight therapeutic categories. Approval success rates were calculated for rDNA therapeutics with two possible fates: (i) approval in any country and (ii) U.S. approval only. Global approval success rates ranged from $23 \%$ to $63 \%$, and U.S. approval success rates ranged from $17 \%$ to $58 \%$. Trends in clinical phase lengths over five time periods and an overview of the rDNA therapeutics currently under Food and Drug Administration review are discussed.
\end{abstract}

\section{INTRODUCTION}

The clinical development of novel therapeutics, including recombinant proteins (rDNAs), is commonly divided into three phases (i.e., phases I, II, and III). Each phase is more complex, time-consuming, and resource-intensive than the preceding one. Initial testing of new therapeutics in humans occurs in phase I. The objectives of this phase include the determination of initial safety and pharmacokinetic and pharmacodynamic profiles for the product (e.g., reported adverse effects, highest tolerated dose, and rates of absorption, metabolism, and distribution of product in the body after various doses). Testing may occur in either normal volunteers or in patients. If the product fares well in phase I, then phase II studies are conducted to determine product efficacy in patients and to provide additional safety information. Data from these pilot studies are used to design the pivotal phase III studies, which provide precise measurements of safety and efficacy of the product in a targeted patient population. The exact indication the Food and Drug Administration (FDA) or other regulatory agency will consider for approval is determined by the phase III study data.

Clinical development programs must be tailored to the product. The time and resources required to perform the clinical studies can vary according to many factors, including the type and number of indications studied, the number of studies and subjects involved, the complexity of the study protocol, the availability of appropriate study subjects, and the number and location (e.g., single country or multiple countries) of study sites (1). Limited data on clinical development pro- grams of rDNA therapeutics are available; the data show that the range in the total number of studies (4-23 studies) and total number of subjects (390-22,270 subjects) involved in the programs is highly variable (2).

The success of clinical development programs can certainly be influenced by the factors noted above, but it can also be affected by less tangible considerations such as significant or rapid technological change, the economic viability of the sponsoring company, the previous experience of the sponsoring company in the clinical development of therapeutics, changing regulatory requirements, and the existence of competitive products either in development or on the market. Success can be measured by tracking the advancement of products through the clinical phase and review transitions to marketing approval. Using this method, the success of the biopharmaceutical industry's efforts to develop and launch rDNA therapeutics in various therapeutic categories for the U.S. and global markets can be quantified. In this report, clinical phase and review transition probabilities, as well as approval success rates, for eight therapeutic categories are presented. Clinical phase lengths over five time periods and an overview of the rDNA therapeutics currently undergoing FDA review are discussed.

\section{ANALYSIS CRITERIA}

The Tufts Center for the Study of Drug Development collected clinical development and regulatory review data for 271 rDNA therapeutics that entered clinical study during 
1980-2002 from company surveys and public documents. Therapeutic monoclonal antibodies, rDNA products developed as "therapeutic vaccines," and variants of existing products (e.g., reformulations or new doses) were excluded. Of the 271 products, 222 originated at or entered clinical studies sponsored by 59 U.S.-based companies. The remaining 49 products originated from the 10 multinational companies that received FDA marketing approval for one or more therapeutic rDNA products.

Data were stratified in various ways for the calculation of phase transition probabilities, approval success rates, and clinical phase lengths. Clinical phase transitions (phase I to II and phase II to III) and regulatory review transitions (phase III to review and review to approval) noted by May 1, 2003 were included. Studies identified as phase I/II were classified as phase II, and phase II/III studies were classified as phase III for the purposes of these analyses. Review transitions were defined for products with two possible fates: $(i)$ any approval and (ii) U.S. approval only. Products in regulatory review or approved in countries other than the U.S. were assigned to phase III for the calculation of phase transitions for the U.S.approval-only cohort, based on the assumption that the sponsoring company might seek U.S. approval in the future. The primary therapeutic category assigned was, in descending priority order, the category of first approval, the category studied in most advanced phase, or the category in which the majority of advanced phase studies were done.

\section{PHASE TRANSITIONS AND APPROVAL SUCCESS RATES FOR rDNA THERAPEUTICS}

The probabilities for clinical phase and regulatory review transitions leading to possible approval in any country for the cohort of all rDNA therapeutics were as follows: $89 \%$ for phase I to II, $69 \%$ for phase II to III, $72 \%$ for phase III to any review, and $96 \%$ for any review to approval. Variations were observed when the data were stratified according to the assigned primary therapeutic category (Figure 1). The transition probabilities for five of the eight therapeutic categories followed the same general trend: the probability of phase I to II transition was high $(80 \%-95 \%)$, decreased at the phase II to III transition $(50 \%-70 \%)$, then increased slightly at the phase III to review transition (65\%-90\%), and was highest at the review to approval transition $(95 \%-100 \%)$.

Products developed for two of the therapeutic categories (endocrine and stimulation of blood cell production) showed notable exceptions to the general trend in transition probabilities (i.e., for both therapeutic categories, either the phase II to III transition was higher than expected or the phase III to review transition probability was lower than expected). The phase II to III transition probability for rDNA therapeutics developed as endocrine treatments was exceptionally high $(94 \%)$. The majority of the products in this category were rDNA versions of well-characterized and well-studied biological proteins (e.g., insulin, human growth hormone, glucagon,

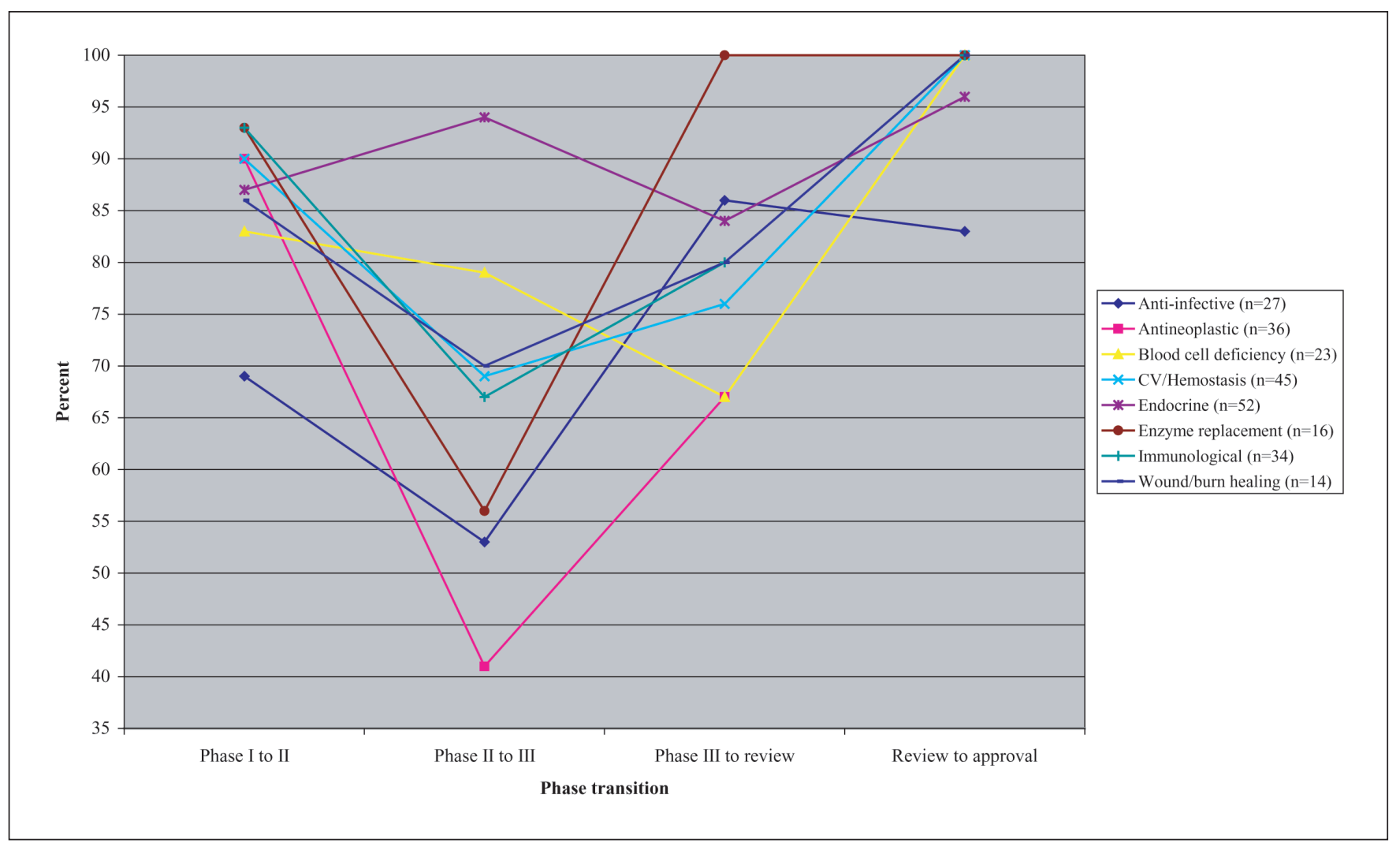

Figure 1. Probabilities for clinical and any review phase transitions for therapeutic recombinant proteins. CV, cardiovascular. Phase transition probabilities were calculated as follows: the number of products that completed a given phase (e.g., phase I) and entered the next phase (e.g., phase II) divided by the total number of products that entered the phase but did not remain (i.e., all products entering the phase minus those that remained in that phase of clinical development). 
Table 3. Therapeutic Recombinant Proteins Approved by the FDA in 2003 or Under FDA Review

\begin{tabular}{|c|c|c|c|c|}
\hline $\begin{array}{l}\text { Sponsoring } \\
\text { Company }\end{array}$ & Generic Name & $\begin{array}{l}\text { U.S. } \\
\text { Trade Name }\end{array}$ & Review and Approval Status & Therapeutic Category \\
\hline Biogen & Alefacept & Amevive & FDA approved on $01 / 31 / 2003$ & Immunological \\
\hline Pharmacia & Pegvisomant & Somavert & FDA approved on $03 / 25 / 2003$ & Endocrine \\
\hline Genzyme & Agalsidase $\beta$ & Fabrazyme & $\begin{array}{l}\text { FDA approved on 04/24/2003; } \\
\text { E.U. and other non-U.S. approvals }\end{array}$ & Enzyme replacement \\
\hline $\begin{array}{l}\text { BioMarin } \\
\text { Pharmaceutical }\end{array}$ & Laronidase & Aldurazyme & $\begin{array}{l}\text { FDA approved 4/31/2003; } \\
\text { EMEA review }\end{array}$ & Enzyme replacement \\
\hline $\begin{array}{l}\text { Transkaryotic } \\
\text { Therapies }\end{array}$ & Epoetin $\Delta$ & Dynepo & FDA review; approved in E.U. & Blood cell deficiency \\
\hline Novo Nordisk A/S & Insulin detemir & & FDA and EMEA review & Endocrine \\
\hline $\begin{array}{l}\text { Unigene } \\
\text { Laboratories }\end{array}$ & Calcitonin (salmon) & Fortical & $\begin{array}{l}\text { FDA review; approved in E.U., } \\
\text { Switzerland }\end{array}$ & Endocrine \\
\hline $\begin{array}{l}\text { Transkaryotic } \\
\text { Therapies }\end{array}$ & Agalsidase $\alpha$ & Replagal & $\begin{array}{l}\text { FDA review; E.U. and other } \\
\text { non-U.S. approvals }\end{array}$ & Enzyme replacement \\
\hline
\end{tabular}

(aldesleukin and denileukin diftitox) are approved for marketing in the U.S. Three of these four started in clinical studies earlier than, or during the same year as, clinical study initiation of the products that later failed at phase II. Thus, the decision to discontinue other antineoplastic interferon and interleukin products at phase II might have been based, in part, on the identification of competitive products that were further along in clinical development.

The phase III to review transition probability for rDNA therapeutics developed as enzyme replacement therapies was exceptionally high $(100 \%)$. These products have biological counterparts with known function (e.g., glucocerebrosidase, $\alpha$-glucosidase, and $\alpha$-galactosidase A). Since the rDNA versions simply replace the missing biological ones, there is less risk associated with the clinical development of these proteins compared to that for novel rDNA therapeutics. Difficulties in development can still be encountered, most notably in devising ways to deliver adequate quantities of the replacement rDNA product to the desired location in the body. Once these products have demonstrated preliminary safety and efficacy in phase II, the transitions from phase III through approval have been smooth.

Table 1 shows probabilities for clinical phase and regulatory review transitions in the U.S. Approval success rates for rDNA therapeutics with two possible fates, (i) U.S. approval and (ii) approval in any country, are also presented. The probabilities for phase I to II and phase II to III transitions are identical to those shown in Figure 1. In comparison with Figure 1, the remaining transition probabilities and the approval success rates in Table 1 for any given therapeutic category are similar. However, one therapeutic category had notable differences in some values. The blood cell production stimulants have a lower phase III to U.S. review transition probability (50\%), compared to the phase III to any review value (67\%). Moreover, this same category of products has a lower U.S. approval success rate (23\%), compared to the global approval success rate (43\%).

\section{FDA-APPROVED rDNA THERAPEUTICS: CLINICAL} DEVELOPMENT IN FIVE TIME PERIODS

Table 2 shows clinical phase lengths for approved rDNA therapeutics developed over the course of five time periods. Phase lengths were calculated with data stratified in two ways: $(i)$ by the year of FDA approval and (ii) by the year of clinical study initiation. With data stratified by year of FDA approval, both the mean and median clinical phase lengths show a steady increase over time. The advantage of using this method to calculate clinical phase lengths is that products are identified and cohorts are completed by the end of any given year. However, the data are "left-censored" (i.e., since clinical study of commercially sponsored rDNA products did not start until 1980, products approved between 1985 and 1989 had a maximum of 10 years for clinical study, whereas products approved between 2000 and 2002 had a maximum of 23 years for clinical study). In theory, the left-censored feature of the data could result in shorter calculated values for the earlier time periods. In reality, the calculated phase lengths for all five time periods are much shorter than can be explained by this effect; the mean clinical phase was only 2.3 years for rDNA therapeutics approved during the period 1985-1989.

The alternative method of analysis involves stratifying data by the year clinical study was initiated. In this method, the data are "right-censored" (i.e., products entering clinical study between 1980 and 1989 had a maximum of 23 years for clinical study, whereas products entering clinical study be- 
tween 1995 and 1999 had a maximum of eight years for clinical study). Despite this, the results still show an increase in clinical phase length between the 1980s and the 1990s. The disadvantage of using this method is that calculated clinical phase lengths for recent time periods are probably not accurate because the fates of all the products in the cohort are not known. For example, only about $50 \%$ of the products with clinical initiation years between 1995 and 1999 have known fates (discontinued or approved). The remaining products (approximately 30) are either in clinical study or regulatory review, and so all have the potential to be approved. Each new approval could change the clinical phase length calculated for the 1995-1999 cohort, so no conclusions can be drawn from the value currently calculated.

\section{RDNA THERAPEUTICS APPROVED BY FDA IN 2003 OR UNDER FDA REVIEW}

From 1982 to 2002, a total of 54 rDNA therapeutics were approved in the U.S. (3). An additional four products were approved between January 1 and May 1, 2003 (Table 3). The rDNA therapeutic most recently approved is laronidase (BioMarin Pharmaceutical, Novato, CA, USA). This product is as a treatment for mucopolysaccharidosis I (MPS I), a progressive, serious, and life-threatening genetic disease characterized by the accumulation of complex carbohydrates in the lysosomes of cells. This accumulation is due to a deficiency of functional $\alpha$-L-iduronidase, a 628-amino-acid enzyme. There are three categories of MPS I: mild (Scheie's syndrome), intermediate (Hurler-Scheie syndrome), and severe (Hurler's syndrome). The range in severity of the disease is due to the effects of different mutations in patients' $\alpha$-Liduronidase gene on the functionality of translated enzyme (4). Enzyme replacement therapy has been shown to reduce liver size and provide some clinical benefit to patients (5).

Four rDNA therapeutics are currently undergoing FDA review and might be approved in the near future. One product is a blood cell production stimulant, two are endocrine therapeutics, and one is a replacement enzyme. The blood cell production stimulant, epoetin $\Delta$ (Transkaryotic Therapies, Cambridge, MA, USA), is a 165-amino-acid human glycoprotein (erythropoietin) produced from a human cell line using gene activation technology. It was approved in March 2002 for marketing in the European Union (E.U.) for treatment of anemia in patients with chronic renal failure, though launch of the product has been delayed because of patent litigation in the UK. If approved by the FDA, U.S. launch might also be delayed by related patent litigation.

Injectable salmon calcitonin (Unigene Laboratories, Fairfield, NJ, USA) is approved in the E.U. for treatment of Paget's disease and hypercalcemia of malignancy, and in Switzerland for osteoporosis. Calcitonin is a single-chain, 32-amino-acid hormone that inhibits bone resorption. The product is produced in a two-step process comprising production of a glycine-extended precursor peptide in Escherichia coli, followed by in vitro posttranslational modification by an amidating enzyme.

Insulin detemir (Novo Nordisk A/S, Bagsvaerd, Denmark) 
is a soluble, long-lasting basal insulin analog for treatment of type 1 and type 2 diabetes. This product is designed to bind reversibly to albumin, which acts as a "depot" for the insulin. A clinical study of insulin detemir has shown that the product "appears to provide more predictable fasting blood glucose levels with lower intra-subject variation and to induce fewer hypoglycemic incidents" (6) compared with a basal insulin currently available, neutral protamine Hagedorn (NPH).

Agalsidase $\alpha$ (Transkaryotic Therapies) is undergoing regulatory review as a replacement therapy for treatment of Fabry disease, an X-linked genetic disorder caused by deficiency of functional $\alpha$-galactosidase A. This 398-amino-acid enzyme catalyzes the hydrolysis of glycosphingolipid and is produced in a human cell line (7). In April 2003, the FDA-approved agalsidase $\beta$ (Genzyme, Cambridge, MA, USA), which is produced in Chinese hamster ovary cells, for the treatment of Fabry disease (8). Both products were designated as orphan drugs in the U.S. Under the U.S. Orphan Drug Act, an orphan therapeutic is given 7 years of U.S. market exclusivity, though another product might subsequently be approved for the same indication if the two products are not considered the same or clinical superiority is demonstrated (9). Agalsidase $\alpha$ and agalsidase $\beta$ were approved simultaneously in the E.U. in August 2001 with co-orphan drug status, which provides E.U. market exclusivity for 10 years.

\section{DISCUSSION}

Phase transition probabilities, approval success rates, and clinical phase lengths are important benchmarks of the process used to get new rDNA therapeutics to the patients who need them. The general trend in the transition probabilities presented here for rDNA therapeutics has also been observed for chimeric and humanized therapeutic monoclonal antibodies (mAbs) (10) and new chemical entities (NCEs) (11). In the case of all three (rDNA, mAbs, and NCEs), the rationale for the trend is the same: preclinical testing should have excluded unsafe products and identified those with the desired pharmacokinetic properties. Therefore, the phase I to II transition probability should be high.

The availability and applicability of preclinical models for human disease states is variable, thus preclinical efficacy data might not be predictive of the efficacy observed in humans in phase II. Therefore, the transition probability for phase II to III decreases. Products that do not show promise at phase II should be discontinued at that point. Greater losses of both time and resources accrue when products fail at either phase III or in regulatory review (11). If a product has demonstrated good safety and efficacy profiles in phases I and II, then, in theory, these results should be duplicated in the larger phase III studies. Therefore, the general trend is that the probability for the phase III to review transition increases. Finally, the safety and efficacy data generated in well-designed and documented studies submitted in highquality marketing applications should be compelling to the regulatory authorities. In theory, the transition probability for review to approval should be $100 \%$.

The approval success rates for rDNA therapeutics vary greatly depending on the therapeutic category. One reason for the higher success rates in the endocrine, immunological, and enzyme replacement categories is that these categories include a greater percentage of rDNA therapeutics that are either identical or analogous to well-studied, therapeutic biological proteins. In contrast, development of novel rDNA proteins or rDNA therapeutics with poorly understood function holds greater risk. Therapeutic categories that included a high percentage of these products had lower phase transition probabilities and approval success rates.

Differences between global and U.S. review transition probabilities and approval success rates are partially due to the fact that, for some products, there is a lag between approval outside as compared to within the U.S. Thus, the U.S. rates will be lower until such time as these products are reviewed and approved in the U.S. Lags in the approval of these products might simply be due to business decisions to pursue other markets first (e.g., the presence of established competitive product in U.S. might initially make overseas markets more attractive). However, at any given time, some products might be blocked from access to the U.S. market because of patent disputes or legal obstacles (e.g., orphan exclusivity provisions for competitor's products).

On average, the time taken to perform clinical studies of rDNA therapeutics increased between the 1980s and the 1990s. This trend is probably related to the reason some approval success rates are high: rDNA versions of biological proteins with known therapeutic value are more likely to be successful and have short clinical phases because of the availability of data for the biological counterparts. These products were approved in the 1980s, when biopharmaceutical firms focused on the development of rDNA products thought most likely to succeed. Therefore, the mean clinical phase times for products approved in the 1980s are shorter compared to those approved in the 1990s. Moreover, lengthening clinical phases might be due to the significant technical advances in science and medicine made in the past two decades that, in part, afforded the industry the ability to generate innovative new recombinant therapeutics and study them in humans. These advances also allow the industry to be held to higher standards in areas of FDA oversight (e.g., production, characterization, and clinical study of rDNA therapeutics).

In the literal sense, phase transition probabilities and approval success rates measure the movement of therapeutics through the clinical development and approval process. They are also a measure of how efficiently industry develops innovative products (e.g., how effectively resources are allocated). The robust approval success rates presented here suggest that recombinant technology will continue to be a significant source of new therapeutics in the future. This, coupled with the fact that nearly 80 rDNA therapeutics have been approved worldwide, suggests that the biopharmaceutical industry has successfully exploited biotechnology to produce innovative products that benefit patients.

\section{ACKNOWLEDGMENTS}

The authors thank the companies that provided data for 
this study and the people who completed the survey. We thank Andrew Mulcahy for his able and diligent assistance with the compilation of the data and Dr. Joseph DiMasi for helpful comments and suggestions.

\section{REFERENCES}

1.Spilker, B. 1991. Establishing clinical trial objectives, p. 10-14. In Guide to Clinical Trials. Raven Press, New York.

2.Reichert, J.M. 2001. Clinical development of therapeutic medicines: a biopharmaceutical versus pharmaceutical product comparison. Drug Inf. J. 35:337-346.

3.Reichert, J.M. and C. Paquette. 2003. Therapeutic recombinant proteins: trends in U.S. approvals 1982 to 2002. Curr. Opin. Mol. Ther. 5:139-147.

4.Scott, H.S., S. Bunge, A. Gal, L.A. Clarke, C.P. Morris, and J.J. Hopwood. 1995. Molecular genetics of mucopolysaccharidosis type I: diagnostic, clinical, and biological implications. Hum. Mutat. 6:288-302.

5.Kakkis, E.D., J. Muenzer, G.E. Tiller, L. Waber, J. Belmont, M. Passage, B. Izykowski, J. Phillips, et al. 2001. Enzyme-replacement therapy in mucopolysaccharidosis I. N. Engl. J. Med. 344:182-188.

6.Hermansen, K., S. Madsbad, H. Perrild, A. Kristensen, and M. Axelsen. 2001. Comparison of the soluble basal insulin analog insulin detemir with NPH insulin: a randomized open crossover trial in type 1 diabetic subjects on basal-bolus therapy. Diabetes Care 24:296-301.

7.Schiffmann, R., J.B. Kopp, H.A. Austin, III, S. Sabnis, D.F. Moore, T. Weibel, J.E. Balow, and R.O. Brady. 2001. Enzyme replacement therapy in Fabry disease: a randomized controlled trial. JAMA. 285:27432749 .

8.Eng, C.M., N. Guffon, W.R. Wilcox, D.P. Germain, P. Lee, S. Waldek, L. Caplan, G.E. Linthorst, et al. 2001. Safety and efficacy of recombinant human $(\alpha)$-galactosidase A replacement therapy in Fabry's disease. N. Engl. J. Med. 345:9-16.

9.Milne, C.-P. 2002. Orphan products_-pain relief for clinical development headaches. Nat. Biotechnol. 20:780-784.

10.Reichert, J.M. 2001. Monoclonal antibodies in the clinic. Nat. Biotechnol. 19:819-822

11.DiMasi, J.A. 2001. Risks in new drug development: approval success rates for investigational drugs. Clin. Pharmacol. Ther. 69:297-307.

\section{Address correspondence to:}

Janice M. Reichert

Tufts Center for the Study of Drug Development

192 South St., Suite 550

Boston, MA 02111, USA

e-mail: janice.reichert@tufts.edu 